\title{
Observation of optical spring effect in a microtoroidal optomechanical resonator
}

\author{
Mani Hossein-Zadeh* and Kerry J. Vahala \\ Department of Applied Physics, California Institute of Technology, Pasadena, California 91125, USA \\ *Corresponding author: mhz@caltech.edu
}

Received February 6, 2007; revised April 5, 2007; accepted April 7, 2007;

posted April 11, 2007 (Doc. ID 79864); published June 5, 2007

\begin{abstract}
We present experimental evidence of the optical spring effect in a silica microtoroid resonator. The variation of the measured mechanical resonant frequency as a function of optical power, optical coupling, and optical detuning is in very good agreement with a model for radiation-pressure-induced rigidity in a silica microtoroid. (C) 2007 Optical Society of America

OCIS codes: $230.5750,200.4880,190.0190$.
\end{abstract}

It is well known that the large circulating optical power inside an optical resonator can couple the optical and mechanical dynamics of the resonant cavity through radiation pressure [1]. When the wavelength of the optical input power to an optical resonator is detuned from resonance, the radiation pressure creates a restoring force that modifies the effective rigidity and the effective mechanical loss factor of mechanical modes of the resonator structure. If the optical power is large enough, the response of the structural deformation in conjunction with the optical decay (dynamical back action [1]) can result in self-sustained oscillation [2-4] or cooling $[5,6]$ of the mechanical modes (depending on the relative detuning of the pump wavelength with respect to the resonant wavelength). At low optical power the optomechanical coupling manifests itself as modified resonant frequency (due to optically induced rigidity) and reduction of mechanical resonant linewidth [4]. Optically induced rigidity, known as the optical spring effect, has been previously studied in cantilever-based Fabry-Perot resonators [7,8]. However, its observation and characterization can be significantly simplified in microtoroidal resonators owing to their small size, large optical $Q$, and efficient optical coupling mechanism. Although the signature of the optical spring effect has been observed in silica microtoroid resonators $[4,5]$, its behavior has not been fully explored and characterized in these structures. In this Letter we present a simple classical model followed by experimental results that connect the strength of the optical spring effect to the optical and mechanical properties of a silica microtoroid resonator.

In a silica microtoroid optical resonator, the presence of the circulating optical power modifies the mechanical resonant frequency through optical absorption (thermal effect), reduction of the effective loss factor (optomechanical gain effect), and modified rigidity (optical spring effect) [4]. Here we study a case where the optically induced mechanical resonant shift is dominated purely by the optical spring effect. The thermomechanical effect is suppressed by increasing the pillar diameter (stronger heat sink) as well as by choosing a mechanical mode with less sensitivity to thermal gradients within the structure.
Furthermore, the optomechanical gain effect is made negligible by choosing a microtoroid with relatively low mechanical quality factor. When the optical input power is smaller than the threshold power for selfsustained optomechanical oscillations, $P_{\text {th }}$ (see Ref. $[2-4]$ ), the mechanical resonant frequency can be written as

$$
f=f_{0}\left(1+\eta_{\mathrm{os}} P_{\text {in }}\right)
$$

Here $P_{\text {in }}$ is the optical input power, $f_{0}$ is the intrinsic resonant frequency of the mechanical mode, and $\eta_{\mathrm{os}}$ is the optical spring coefficient. In this study we use the first flexural mode of the microtoroid structure [2]. Figure 1 shows that the radial position of the optical mode $(r)$ is coupled to the mechanical motion in the $z$ direction; so the radiation pressure force affects the dynamics of the first mechanical mode. As described previously $[2,3]$, the offset of the toroid with respect to the silica support disk [Fig. 1(a)] is responsible for the optomechanical coupling to the first mode. Also, because of this offset the optical force $\left(F_{\text {opt }}\right)$ generates a nonzero torque equivalent to an effective force along the $z$ axis that can be estimated as $F_{z} \sim \beta \times F_{\text {opt }}$ ( $\beta$ is approximately equal to the ratio between the offset and the mechanical arm).

For small amplitude oscillations the motion of the toroid is mainly along the $z$ axis [Fig. 1(b)]. The dynamic of the passive resonator can then be reduced to that of a one-dimensional harmonic oscillator moving along the $z$ direction with an angular frequency of $\Omega_{0}=2 \pi f_{0}=\left(k_{0} / m_{\mathrm{eff}}\right)^{1 / 2}$, where $m_{\text {eff }}$ and $k_{0}$ are the effective mass and the intrinsic rigidity of the correspond-

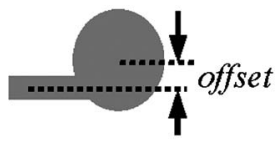

(a)

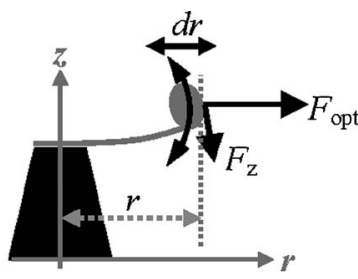

(b)
Fig. 1. (a) Toroid offset with respect to support disk. (b) Coupling of the radiation-pressure-induced force $\left(F_{\mathrm{rad}}\right)$ to the first eigenmechanical mode of the microtoroid. 
ing mechanical mode. The presence of the circulating optical power in the silica microtoroid structure modifies the rigidity of the mechanical mode such that $k=k_{0}+k_{\text {opt }}$. Near an optical resonance the optical rigidity $\left(k_{\text {opt }}\right)$, optical force $\left(F_{\text {opt }}\right)$, and the circulating optical power $\left(P_{\text {circ }}\right)$ are coupled through the following equations:

$$
\begin{gathered}
k_{\text {opt }}=-\frac{\mathrm{d}\left(\beta \times F_{\mathrm{opt}}\right)}{\mathrm{d} z}=-\beta\left(\frac{\mathrm{d} r}{\mathrm{~d} z}\right) \frac{\mathrm{d} F_{\mathrm{opt}}}{\mathrm{d} r}, \\
F_{\text {opt }}=\frac{2 \pi n}{c} P_{\text {circ }}, \\
P_{\text {circ }}=\frac{\lambda P_{\text {in }} Q_{0}}{4 \pi^{2} n R_{0}} \frac{y(1-y)}{d^{2}+0.25},
\end{gathered}
$$

where $y=Q_{\mathrm{L}} / Q_{0}$ and $d=\left(\lambda-\lambda_{0}\right) / \Delta \lambda_{\mathrm{L}}$ is the normalized detuning from the resonant wavelength $\left(\lambda_{0}=2 \pi n r / l\right.$, $l$ is an integer). $\lambda$ is the laser wavelength, $c$ is the speed of light, $n$ is the effective refractive index of the optical mode, and $R_{0}$ is the radius of the microtoroid. $Q_{\mathrm{L}}$ and $Q_{0}$ are the loaded and intrinsic optical quality factors of the resonator, respectively. So $y$ quantifies the optical coupling strength between the waveguide and the microresonator $(y>0.5$ undercoupled, $y$ $<0.5$ overcoupled, and $y=0.5$ critically coupled). For small mechanical frequency shifts $\left(\Omega_{0} \gg \Omega-\Omega_{0}\right.$, as in the case of radiation-pressure-induced shift) the mechanical resonant frequency is estimated as $\Omega \approx \Omega_{0}$ $+\left(k_{\text {opt }} / 2 m_{\text {eff }} \Omega_{0}\right)$. From Eqs. (1) and (2a)-(2c), the optical spring coefficient $\left(\eta_{\mathrm{os}}\right)$ can be estimated as

$$
\eta_{\mathrm{os}}=\frac{\beta(\mathrm{d} r / \mathrm{d} z)}{\Omega_{0}^{2} m_{\mathrm{eff}} \omega_{0} R_{0}^{2}} Q_{0}^{2}\left(y^{2}-y^{3}\right) d\left(d^{2}+0.25\right)^{-2} .
$$

This equation describes the behavior of the frequency shift as a function of normalized wavelength $(d)$, coupling strength $(y)$, and the intrinsic characteristics of the resonator. According to this equation $\eta_{\mathrm{os}}$ scales as $\left(1 / \Omega_{0}^{2}\right)$; so selection of a low-frequency mechanical mode is desirable to boost the optical spring effect. Note that, as opposed to self-sustained optomechanical oscillation and optomechanical cooling, optical spring is an instantaneous effect and therefore does not depend on the mechanical quality factor $\left(Q_{\text {mech }}\right)$.

Figure 2(a) shows a top-view photograph of the

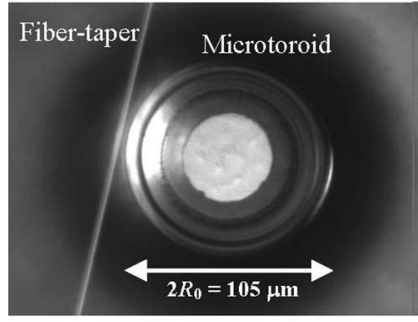

(a)

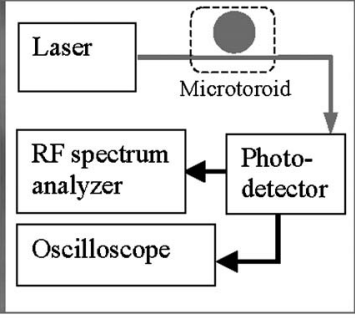

(b)
Fig. 2. (a) Top-view photograph of the silica microtoroid coupled to fiber taper. (b) Schematic diagram of the experimental configuration. silica microtoroid used in this work. The output of a tunable laser is coupled to the optical modes of the microtoroid by using a fiber taper [shown in Fig. 2(a)]. The transmitted optical power is detected by a photodetector, and the photocurrent is analyzed by using an RF spectrum analyzer. The oscilloscope monitors the optical mode and the detuning from the resonant wavelength $(d)$. In our experiment the optical input power $\left(P_{\text {in }}\right)$ is always about five times smaller than $P_{\text {th }}$; so the mechanical resonator is primarily thermally driven. Figure 3(a) shows the RF spectrum of the detected optical power. This spectrum is the result of thermal fluctuations of the mechanical mode imprinting onto the optical probe power. Using finite element modeling (FEMLAB software), we have calculated the first three eigenmechanical modes of the microtoroid that match the measured values (the gray dots). The splitting of the measured frequencies is due to imperfections in the silicon pillar that break its cylindrical symmetry.

As noted above, the first eigenmode is used in this work. This mode has a calculated eigenfrequency of about $2.4 \mathrm{MHz}$ and intrinsic rigidity of $k_{0}=9.5$ $\times 10^{3} \mathrm{~N} \mathrm{~m}^{-1}$. Figure $3(\mathrm{~b})$ shows the cross-sectional diagram of the microtoroid structure and the deformation associated with the first eigenmechanical mode. The measured quality factor $\left(Q_{\text {mech }}\right)$ for this mode is about 250 . Using finite element modeling, we have also calculated $m_{\text {eff }}\left(4.18 \times 10^{-11} \mathrm{Kg}\right), \mathrm{d} r / \mathrm{d} z$ $(\sim 0.057)$, and $\beta(\sim 0.09)$. The mechanical resonant frequency of the first eigenmode measured for six different optical powers and at a fixed detuning ( $d$ $=0.5)$ is given in Fig. 4(a). The linear fit is used to evaluate the intrinsic mechanical resonant frequency $\left(f_{0=} \Omega_{0} / 2 \pi=2373.6 \mathrm{KHz}\right)$ as well as the optical spring coefficient $\left(\eta_{\mathrm{os}}=1.78 \mathrm{~W}^{-1}\right)$. The ratio of the radiation pressure induced and the intrinsic rigidity can be estimated as $k_{\text {opt }} / k_{0} \approx 2 \times \eta_{\text {os }} \times P_{\text {in }}\left(k_{\text {opt }} / k_{0} \approx 1.8 \times 10^{-3}\right.$ for $\left.P_{\text {in }}=500 \mu \mathrm{W}\right)$.

Figure 4(b) shows the measured value of $\eta_{\mathrm{os}}$ at different optical detunings $(d)$ and for three different optical input powers. The solid line is the theoretical prediction using Eq. (3) and the simulated values of $m_{\text {eff }}, \mathrm{d} r / \mathrm{d} z$, and $\beta$. The laser is tuned to an optical mode with a intrinsic quality factor of $Q_{0}=6.5 \times 10^{6}$,

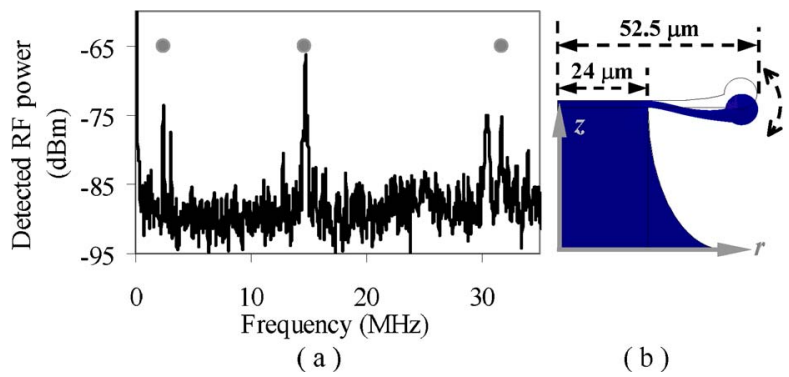

Fig. 3. (Color online) (a) Noise spectra of the eigenmechanical modes of the microtoroidal cavity in the RF spectrum of the detected optical power. The gray dots are the simulated values of the first three eigenfrequencies using finite element modeling (FEMLAB). (b) Cross-sectional diagram of the microtoroid structure and deformation associated with the first eigenmechanical mode. 


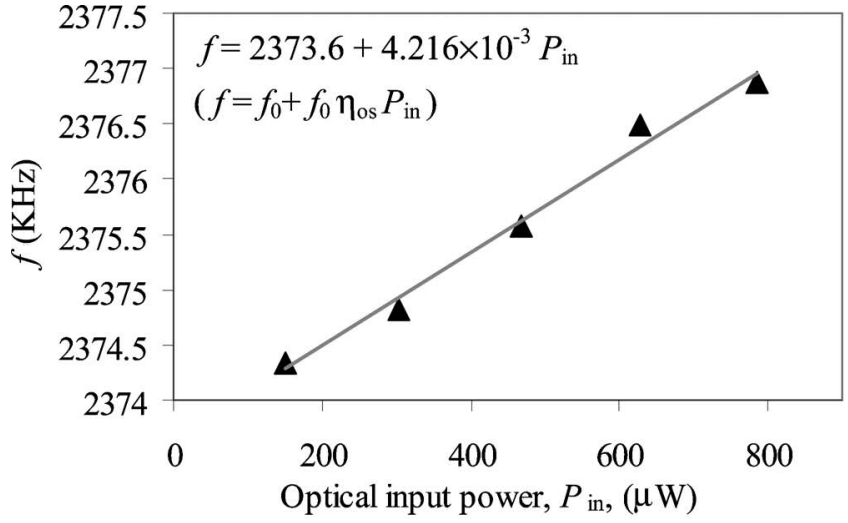

(a)

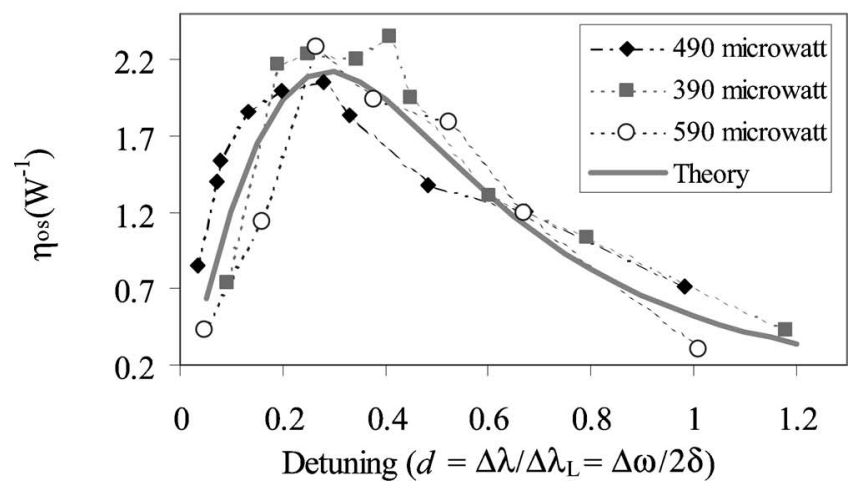

(b)

Fig. 4. (a) Measured mechanical resonant frequency plotted against optical input power at critical coupling $\left(Q_{\mathrm{L}} / Q_{0}=0.5\right)$ and $d=0.5$. The solid line is a linear fit to the experimental data. (b) Measured value of $\eta_{\mathrm{os}}$ plotted against optical detuning $(d)$ for three different optical input powers $\left(P_{\text {in }}\right)$ at critical coupling $\left(Q_{L} / Q_{0}=0.5\right)$. The solid curve is the theoretical prediction based on Eq. (3). $Q_{0}$ is about $6.5 \times 10^{6}$ for both measurements.

and the microtoroid is critically coupled to the fiber taper $\left(Q_{\mathrm{L}} / Q_{0}=0.5\right)$. The good agreement between the theoretical prediction and the measured values shows that the frequency shift is indeed dominated by the radiation-pressure-induced optical spring effect. As a further test of the validity of the theoretical model we have measured $\eta_{\mathrm{os}}$ as a function of optical coupling strength $\left(Q_{\mathrm{L}} / Q_{0}\right)$. $Q_{\mathrm{L}}$ is tuned and measured by controlling the coupling gap (between the fiber taper and the microtoroid) and monitoring the transmitted optical power.

Figure 5 shows the measured values of $\eta_{\text {os }}$ plotted against $Q_{\mathrm{L}} / Q_{0}$ while $d=0.5$ and the laser is tuned to an optical mode with $Q_{0}=1.2 \times 10^{7}$. The measured values are in relatively good agreement with the theoretical prediction from Eq. (3) (solid curve). Note that at $Q_{\mathrm{L}} / Q_{0}=0.5$ (critical coupling) $\eta_{\mathrm{os}}$ is about 3.37 times larger than its value at $d=0.5$ in the detuning experiment $\left(5.4 \mathrm{~W}^{-1}\right.$ in Fig. 5 as opposed to $1.6 \mathrm{~W}^{-1}$ in Fig. 4). This is also in good agreement with the theoretical model, since, according to Eq. (3), $\eta_{\mathrm{os}}$ is proportional to $Q_{0}^{2}$ and $Q_{0}^{2}$ is 3.41 times larger for the data in Fig. 5 than that in Fig. $4\left(1.2^{2} \times 10^{14}\right.$ as op-

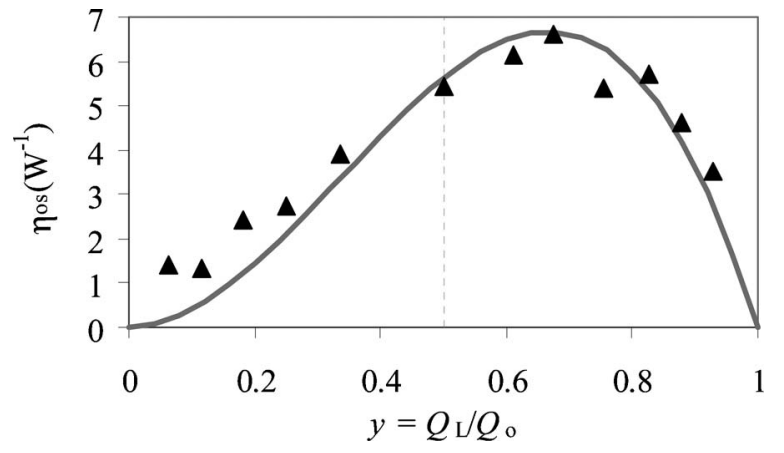

Fig. 5. Measured value of $\eta_{\mathrm{os}}$ plotted against $Q_{\mathrm{L}} / Q_{0}$ at $d$ $=0.5$ for an optical mode with $Q_{0}=1.2 \times 10^{7}$. The solid curve is the theoretical prediction based on Eq. (3).

posed to $0.65^{2} \times 10^{14}$ ). In all experiments the laser wavelength is blueshifted relative to the optical resonance $(d>0)$. Due to the strong thermal instability in high- $Q$ systems, the redshifted regime $(d>0)$ is accessible only by using a fast tunable laser and ultrafast electronic locking system [5].

In conclusion, we have characterized the optical spring effect in a microtoroidal optomechanical resonator. A closed-form equation is derived based on a simple theory that accurately predicts the experimental outcomes. Observation and characterization of this effect is an important step toward using these structures for studying quantum optical phenomena that are related to radiation-pressure-induced rigidity, such as ponderomotive squeezing of light and radiation-pressure-assisted quantum entanglement $[9,10]$.

This work was supported by the Caltech Lee Center for Advanced Networking and the Caltech Center for the Physics of Information.

\section{References}

1. V. B. Braginsky and S. P. Vyatchanin, Phys. Lett. A 293, 228 (2002).

2. T. J. Kippenberg, H. Rokhsari, T. Carmon, A. Scherer, and K. J. Vahala, Phys. Rev. Lett. 95, 033901 (2005).

3. H. Rokhsari, T. J. Kippenberg, T. Carmon, and K. J. Vahala, IEEE J. Sel. Top. Quantum Electron. 12, 96 (2006).

4. M. Hossein-Zadeh, H. Rokhsari, A. Hajimiri, and K. J. Vahala, Phys. Rev. A 74, 023813 (2006).

5. A. Schliesser, P. Del'Haye, N. Nooshi, K. J. Vahala, and T. J. Kippenberg, Phys. Rev. Lett. 97, 243905 (2006).

6. S. Gigan, H. R. Bohm, M. Paternostro, F. Blaser, G. Langer, J. B. Hertzberg, K. C. Schwab, D. Bauerle, M. Aspelmeyer, and A. Zeilinger, Nature 444, 67 (2006).

7. T. Corbitt, Y. Chen, F. Khalili, D. Ottaway, S. Vyatchanin, S. Whitcomb, and N. Mavalvala, Phys. Rev. A 73, 023801 (2006).

8. B. S. Sheard, M. B. Gray, C. M. Mow-Lowry, D. E. McClelland, and S. E. Whitcomb, Phys. Rev. A 69, 051801(R) (2004).

9. S. W. Schediwy, C. Zhao, L. Ju, and D. G. Blair, Class. Quantum Grav. 21, 1253 (2004).

10. S. Mancini, V. Giovannetti, D. Vitali, and P. Tombesi, Phys. Rev. Lett. 88, 120401 (2002). 